\title{
Aptamer-Based Precipitation as an Alternative to the Conventional Immunoprecipitation for Purification of Target Proteins
}

\author{
Seongeun Song, Yea Seul Cho, Sung-Jae Lee, ${ }^{\dagger}$ and Sang Soo Hah ${ }^{*}$ \\ Department of Chemistry and Research Institute for Basic Sciences, Kyung Hee University, Seoul 130-701, Korea \\ *E-mail:sshah@khu.ac.kr \\ †Department of Biology, Kyung Hee University, Seoul 130-701, Korea \\ Received April 29, 2014, Accepted May 8, 2014
}

\begin{abstract}
Aptamers are oligonucleotides or peptide molecules that are able to bind to their specific target molecules with high affinity via molecular recognition. In this study, we present development of aptamer-based precipitation assays (or simply aptamoprecipitation) for His-tagged proteins and thrombin to compare their purification efficiency with other conventional affinity precipitation methods. A crosslinking method was employed to immobilize thiol-functionalized aptamers onto the surface of polystyrene resins, enabling them to specifically bind to His-tag and to thrombin, respectively. The resulting aptamer-functionalized resins were successfully applied via a one-step experiment to purification of His-tagged proteins from complex E. coli and to thrombin extraction, exhibiting superior or at least comparable purification results to the conventional immobilized metal affinity precipitation or immunoprecipitation.
\end{abstract}

Key Words : Aptamer, His-tagged, Affinity precipitation, Immunoprecipitation

\section{Introduction}

Aptamers are a special class of nucleic acids or peptide molecules that can selectively bind, with high affinity, to a target molecule. ${ }^{1,2}$ They are able to fold into a well-defined three-dimensional structure, enabling the molecular recognition and specific binding of the corresponding target. Thus, emerging as alternatives to antibodies, a number of aptamers have been found to bind specifically to proteins, ${ }^{3-5}$ and used in many bioanalytical applications, such as for specific detection of proteins, ${ }^{3-5}$ metal ions, ${ }^{6-8}$ and small molecules, ${ }^{9,10}$ and for target-specific delivery. ${ }^{11}$

In particular, it has been of great interest to make use of the unique properties of aptamers as a stationary phase in affinity precipitation, because aptamers offer several advantages over the conventional antibody- or protein-based bioanalytical methods, which include low costs, large-scale production, increased stability against high temperature and extreme $\mathrm{pH}$, long half-life and reversible denaturation. ${ }^{1,2}$ Furthermore, during the aptamer synthesis different modifications can be included to the aptamer at desired positions within the oligonucleotide sequence, allowing for broad applications in studying aptamer structures, mapping aptamerprotein interactions, and in vitro selection of catalytic aptamers. As widely accepted, several 5'-modifications of oligonucleotides enables direct immobilization of the aptamer to a solid support in a controlled orientation, which is advantageous over the conventional antibody immobilization. Importantly, this aptamer immobilization onto a solid support in a controlled manner can enhance binding efficiency, leading to increased specific binding because aptamer functionality is highly dependent on the formation of the correct three-dimensional conformation of the aptamer. Another advantage of aptamers over antibodies is to be able to select aptamers that are active under the desired assay conditions (including buffer composition, $\mathrm{pH}$, and temperature) during the SELEX (systematic evolution of ligands by exponential enrichment), an iterative process of selection and amplification. ${ }^{1}$

Accordingly, aptamers have been indeed used in a number of bioanalytical applications for target-specific detection and purification, including our recent report on development of aptamer-based affinity chromatography. ${ }^{12}$ In the present study, to expand the scope of aptamer-based target detection and/or purification methods, we present development of aptamer-based precipitation for His-tagged proteins and thrombin, which can be compared to the conventional immobilized metal affinity precipitation and immunopreciptation, respectively. Thiol-functionalized aptamers able to specifically bind to His-tag or to thrombin were selected and immobilized using a crosslinking method onto the surface of polystyrene (PS) resins (Scheme 1). The resulting anti-Histag and anti-thrombin aptamer-functionalized resins were applied to purification of His-tagged proteins from complex E. coli cell lysates and to thrombin extraction, respectively.

\section{Experiments}

Chemicals and Materials. Reagents were obtained from commercial suppliers and were used without further purification, and depc-treated deionized water was used for all experiments. RNA-based aptamers for specific binding to His-tagged proteins were synthesized, using the antisense oligonucleotide containing the $\mathrm{T} 7$ promoter sequence at the 3 '-end, as previously described, ${ }^{3,12}$ and the resulting RNA was modified to contain a $5^{\prime}$ thiol group, via an enzymatic 
method for the introduction of 5'-terminal sulfhydryl group at the 5'-termini of RNA molecules according to the literature. $^{3,12}$ Prior to the thiol-functionalized RNA preparation, the 5'-deoxy-5'-thioguanosine-5'-monophosphorothioate (GSMP) was synthesized, ${ }^{3}$ as a substrate for T7 RNA polymerase that requires guanosine for efficient transcription initiation. The in vitro transcription after treatment of alkaline phosphatase was used to incorporate a sulfhydryl moiety to 5'-end of RNA molecule. Anti-thrombin 29-mer DNA of 5'-HS- $\left(\mathrm{CH}_{2}\right)_{6}$-AGTCC GTGGT AGGGC AGGTT GGGGT GACT-3' was obtained from Bioneer (Daejeon, Korea). ${ }^{13,14}$ UV absorbance was measured using Agilent 8453 UV-Visible spectrophotometer. All experiments were performed in triplicate.

Preparation of Aptamer-anchored Resins. Preparation of aptamer-anchored PS resins was slightly modified from the literature procedures. ${ }^{12}$ In brief, a mercaptomethyl polystyrene (mercaptomethyl-PS) resin (mesh: 100-200, $2.0 \mathrm{mmol}$ $S$ per gram resin, Novabiochem) was employed as the solid support. The thiol-functionalized anti-His-tag or anti-thrombin aptamer added to mercaptomethyl-PS was $5 \mathrm{~mol} \%$ of the thiol groups exposed overnight at room temperature to form the disulfide bond with the aptamer. Measurement of the concentration of the aptamer in the supernatant allowed us to estimate the amount of anti-His-tag and anti-thrombin aptamer conjugated with the resins to be approximately 1.11 $\mathrm{nmol} / \mathrm{g}$ resin or $0.259 \mathrm{nmol} / \mathrm{g}$ resin, respectively, i.e. 0.111 $\mathrm{mol} \%$ or $0.0259 \mathrm{~mol} \%$ of amino groups were covalently attached to the aptamer. The resulting aptamer-anchored PS resins were used as stationary phases of aptamer-based precipitation (or simply aptamoprecipitation).

His-tagged Porotein Expression and Purification. Histagged $\beta$-galactosidase ( $\beta$-Gal-His) was obtained according to the literature. ${ }^{12}$ Briefly, $\beta$-Gal-His-encoding plasmids (pRSETa) was purchased from Invitrogen. Transformation of the vector into E. coli BL21 was performed utilizing the transformation and storage solution method. ${ }^{12,15}$

Purification of $\beta$-Gal-His from E. coli cell lysate was performed by adapting the protocols for $\mathrm{Ni}^{2+}$-loaded affinity precipitation column (His SpinTrap, GE Healthcare) as recommended by the manufacturer, as well as the affinity precipitation of $\beta$-Gal-His or thrombin using aptamer-functionalized PS in the same manner. To investigate the functional efficiency of the immobilized aptamers for His-tagged protein purification compared to the conventional affinity precipitation methods, the resins were incubated with the lysate containing $\beta$-Gal-His in $250 \mathrm{mM} \mathrm{NaCl}, 50 \mathrm{mM}$ Tris $\cdot \mathrm{HCl}, 5.0 \mathrm{mM} \mathrm{MgCl}$, $\mathrm{pH}$ 7.4, at room temperature for at least $1 \mathrm{~h}$. And for thrombin extraction, the resins with the anti-thrombin aptamer were incubated with the thrombincontaining solution in $140 \mathrm{mM} \mathrm{NaCl}, 20 \mathrm{mM}$ Tris $\cdot \mathrm{HCl}, 1.0$ $\mathrm{mM} \mathrm{MgCl}_{2}, 5.0 \mathrm{mM} \mathrm{KCl}, \mathrm{pH} 7.4$, at room temperature for at least $4 \mathrm{~h}$. Afterwards, the bound protein was extracted from the resulting resins with $0.4 \mathrm{M}$ sodium acetate, $5 \mathrm{mM}$ EDTA, $7 \mathrm{M}$ urea, $\mathrm{pH} 5.5$, for $\beta$-Gal-His or thrombin purification. Further purification of the protein was performed through vivaspin in PBS buffer using vivaspin 500 (Sartorius,
Germany). All of the binding and extracting steps were performed in a volume of $1 \mathrm{~mL}$. As negative controls, thiolmodified resins were used without prior functionalization with aptamers. To evaluate the purification efficiency obtained from aptamer-modified resins, crude $E$. coli cell lysates were used for the purification of His-tagged proteins, and to evaluate the thrombin recovery, $0.5 \mu \mathrm{g} / \mathrm{mL}$ of the thrombin solution mixed with bovine serum albumin (BSA) was loaded onto the aptamer-functionalized resins.

\section{Results and Discussion}

Rivaling antibodies and retaining structural and synthetic advantages, aptamers have been proven as an emerging class of functional oligonucleotides or peptides for highly specific and sensitive interaction with target molecules. Previously, we reported that the highly specific and sensitive interaction between RNA aptamers targeting His-tagged proteins and the aptamer-based detection system for His-tagged proteins could be developed as an alternative to the conventional Western blots. ${ }^{3}$ By taking advantage of utilizing aptamers in the bioanalytical assays over antibodies, an aptamer-based biochip for specific protein and cell detection and quantitation was also established. ${ }^{5,16}$ In addition, we also reported on development of aptamer-based affinity chromatography. ${ }^{12}$ All of the processes were based on solid materials modified with covalently-bound aptamers, where the covalent attachment might be advantageous over the immobilization of antibodies. In these regards, it was assumed that this advantage could be taken as well for methods development of alternatives to the conventional $\mathrm{Ni}^{2+}$-based affinity precipitation method for His-tagged protein purification as well as to the widely-used immunoprecipitation.

To compare aptamer-based downstream processing directly with the conventional $\mathrm{Ni}^{2+}$-based affinity precipitation and immunoprecipitation, we made use of the highly selective interaction of the RNA aptamer for His-tagged protein purification and of the DNA aptamer for thrombin purification, respectively. As illustrated in Scheme 1 and described in the experimental section, thiol-functionalized aptamers were prepared based on the previous reports, ${ }^{3,12}$ and the aptamers were covalently immobilized onto thiol-functionalized solid support in order to develop aptamer-based affinity precipitation (aptamoprecipitation).

Following the characterization of the aptamer-modified resins, the purification efficiency of aptamer-immobilized resins was tested by using cell lysates of $E$. coli expressing His-tagged $\beta$-galactosidase ( $\beta$-Gal-His) and thrombin solution mixed with BSA, respectively. The different fractions of the purification processes based on the aptamer-functionalized PS resins were analyzed by Bradford assay and SDSPAGE and compared. As clearly demonstrated in Figures 1 and 2, $\beta$-Gal-His and thrombin, respectively, were successfully extracted in high purity through the prepared resins. The purity of the His-tagged protein was determined to be more than $95 \%$ by densitometry (Figure 1), which is superior to the purity obtained from the conventional $\mathrm{Ni}^{2+}$-based 

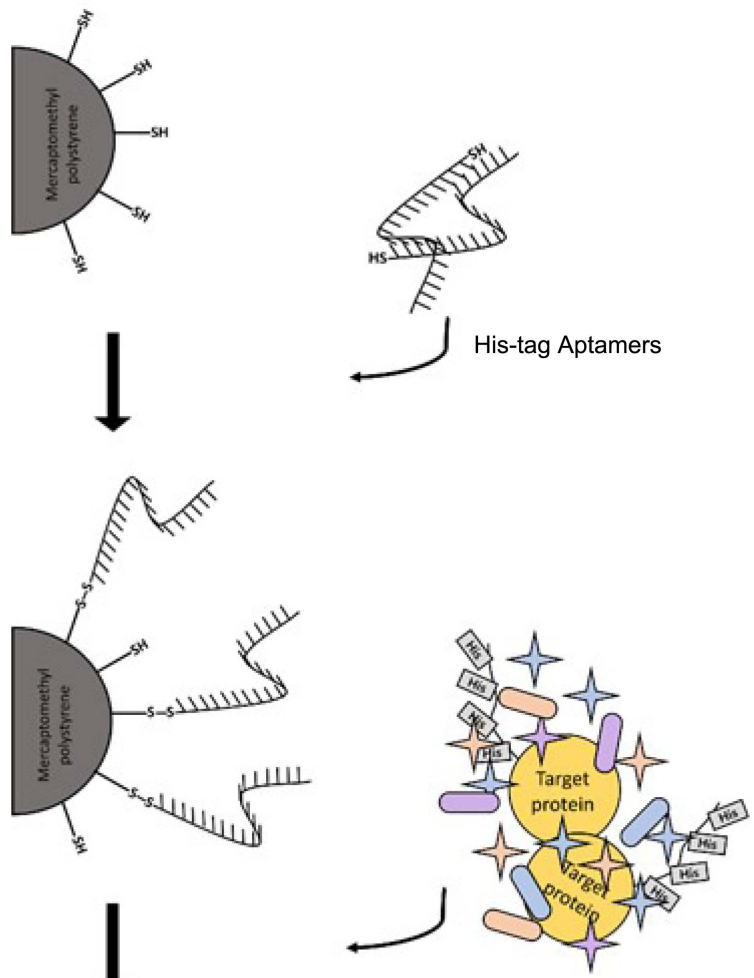

Cell Lysate

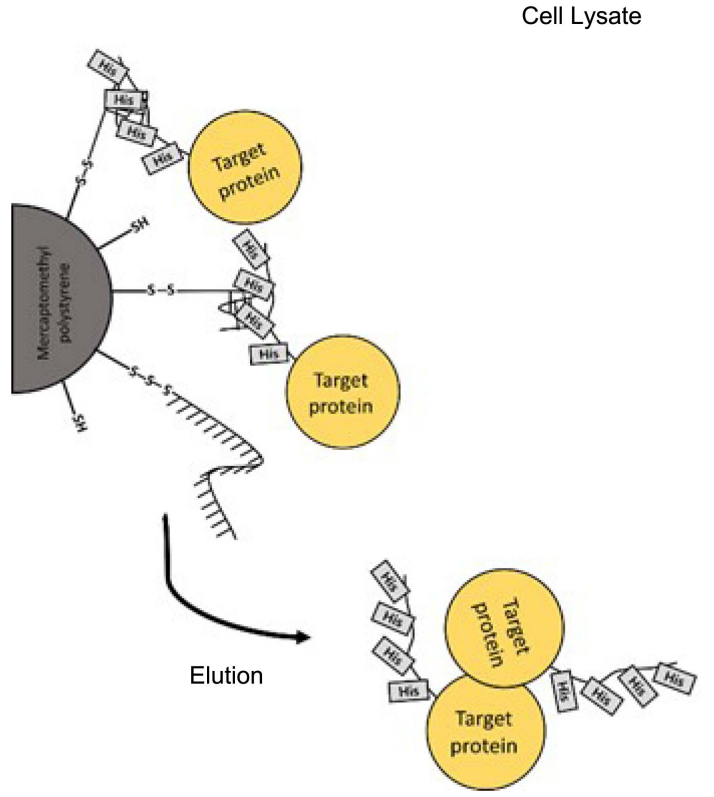

Scheme 1. Schematic representation of aptamer-based precipitation for purification of target protein(s).

precipitation method (unable to be determined). And the thrombin recovery was determined by Bradford assay and densitometry, estimated to be $52 \pm 11 \%$ and $40 \pm 14 \%$, respectively, under the employed conditions (Figure 2). ${ }^{17}$ The relatively low recovery (or purification) yield of thrombin may attribute to the surface density of the aptamerattached resins, which needs to be optimized. Of importance, no BSA was observed in the eluted solution resulting from the thrombin purification experiments, and these results

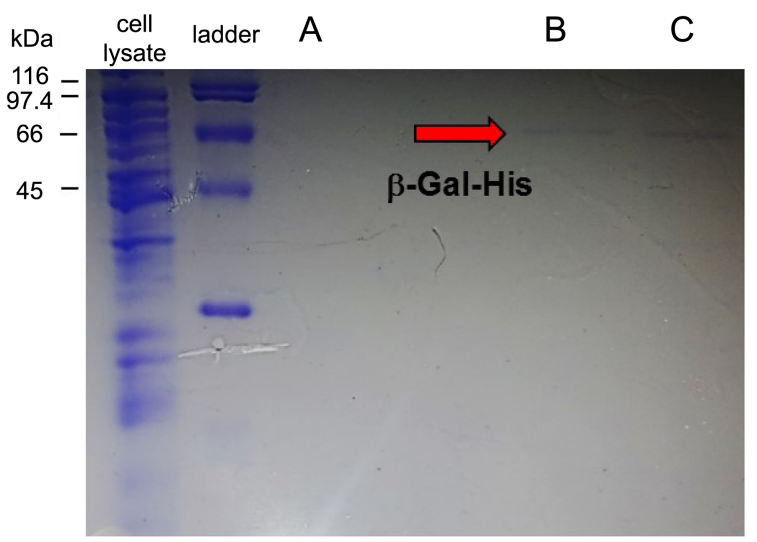

Figure 1. SDS-PAGE analysis of aptamer-based purification of His-tagged $\beta$-galactosidase ( $\beta$-Gal-His). Lane A, extract obtained from the conventional $\mathrm{Ni}^{2+}$-based precipitation; lanes $\mathrm{B}$ and $\mathrm{C}$, extract obtained by using the aptamer-functionalized PS resins for $\beta$-Gal-His precipitation. It should be noted that the loading volume of each extracted fraction was $10 \mathrm{~mL}$, implying that the concentration of $\beta$-Gal-His in the elute resulting from $\mathrm{Ni}^{2+}$-based precipitation was too low to be detected by Coomassie blue staining.

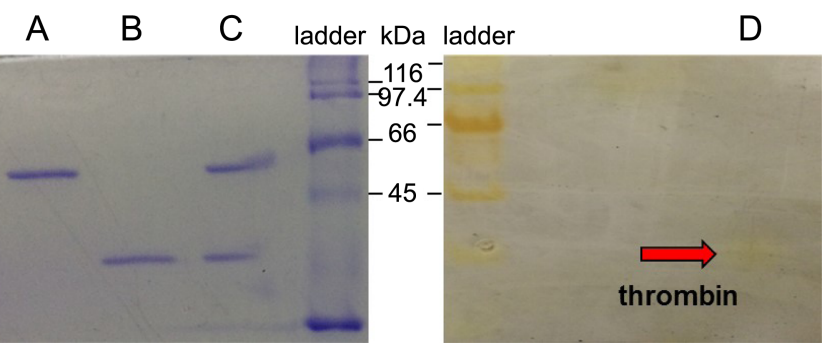

Figure 2. SDS-PAGE analysis of aptamer-based purification of thrombin. Coomassie blue staining and silver staining methods were employed for the left and right gels, respectively. Lane A, bovine serum albumin (BSA) only; $\mathrm{B}$, thrombin only; $\mathrm{C}$ mixture of thrombin and BSA; D, extract obtained by using the aptamerfunctionalized PS resins for thrombin precipitation.

exhibiting high purity of the purified His-tagged protein and no BSA extraction clearly reveal that the target proteins can positively and selectively bind to the immobilized aptamer. Taken together, the purification results are at least comparable to the conventional $\mathrm{Ni}^{2+}$-based affinity precipitation or immunoprecipitation. In addition, due to the existence of disulfide bonds, we expect that the aptamer-conjugated resins or magnetic nanoparticles can be treated with dithiothreitol to remove the conjugated nucleic acids and the new thiol-functionalized resins or magnetic nanoparticles can be recovered, which is cost-effective from an economic point of view. Furthermore, to evaluate the long-term stability of aptamer-modified resins, which is an essential feature of the conventional immunoprecipitation supports, the generated beads could be stored at $4{ }^{\circ} \mathrm{C}$ in binding buffer for different periods of time and the binding of purified $\beta$-GalHis or thrombin $(0.5 \mu \mathrm{g} / \mathrm{mL})$ was investigated after storage for up to 1 month, implying that the aptamer-modified resins could maintain functionality for 1 month. 


\section{Conclusions}

Immunoprecipitation taking advantage of the unique properties of antibodies has been widely utilized as a purification method in various fields in science, especially in biology. However, the conventional immunoprecipitation has several drawbacks, since antibodies are expensive, difficult to selective modification, and unstable at harsh conditions. As an alternative model system, we successfully employed aptamers directed against the His-tag or thrombin in this study, leading to development of an aptamer-based affinity precipitation method for the purification of His-tagged proteins from crude cell lysates or thrombin from thrombin and BSA mixture. The process is based on solid supports modified with covalently bound aptamers, where the covalent attachment may be advantageous over the immobilization of biotinylated antibodies or aptamers to streptavidin-modified supports especially concerning the stability of the solid stationary phase. In addition, compared to the conventional affinity precipitation methods, our study is advantageous because it avoids the utilization of toxic metal ions or of expensive antibodies and the multiplexed separation may be possible by use of different aptamers for various targets. With the recent progresses in the aptamer field, acceleration in the areas of analytical purification and/or quantitation is expected.

Acknowledgments. This work was supported by a grant from the Kyung Hee University in 2013 (KHU-20130549).

\section{References}

1. Nimjee, S. M.; Rusconi, C. P.; Sullenger, B. A. Annu. Rev. Med. 2005, 56, 555-583.

2. Iliuk, A. B.; Hu, L.; Tao, A. Anal. Chem. 2011, 83, 4440-4452.

3. Shin, S.; Kim, I.-H.; Kang, W.; Yang, J. K.; Hah, S. S. Bioorg. Med. Chem. Lett. 2010, 20, 3322-3325.

4. Xiao, Y.; Lubin, A. A.; Heeger, A. J.; Plaxco, K. W. Angew. Chem. Int. Ed. 2005, 44, 5456-5459.

5. Lee, G.-H.; Hah, S. S. Bioorg. Med. Chem. Lett. 2012, 22, 15201522.

6. Ueyama, H.; Takagi, M.; Takenaka, S. J. Am. Chem. Soc. 2002 124, 14286-14287.

7. He, F.; Tang, Y.; Wang, S.; Li, Y.; Zhu, D. J. Am. Chem. Soc. 2005, 127, 12343-12346.

8. Kim, J.; Kim, M. Y.; Kim, H. S.; Hah, S. S. Bioorg. Med. Chem. Lett. 2011, 21, 4020-4022.

9. Liu, J.; Lu, Y. Angew. Chem. Int. Ed. 2006, 45, 90-94.

10. Sankaran, N. B.; Nishizawa, S.; Seino, T.; Yoshimoto, K.; Teramae, N. Angew. Chem. Int. Ed. 2006, 45, 1563-1568.

11. Bagalkot, V.; Farokhzad, O. C.; Langer, R.; Jon, S. Angew. Chem. Int. Ed. 2006, 45, 8149-8152.

12. Lim, H. K.; Kim, I.-H.; Nam, H. Y.; Shin, S.; Hah, S. S. Anal. Lett. 2013, 46, 407-415.

13. Macaya, R. F.; Schultze, P.; Smith, F. W.; Roe, J. A.; Feigon, J. Proc. Natl. Acad. Sci. USA 1993, 90, 3745-3749.

14. Smirnov, I.; Shafer, R. H. Biochemistry 2000, 39, 1462-1468.

15. Chung, C. T.; Niemela, S. L.; Miller, R. H. Proc. Natl. Acad. Sci. USA 1989, 86, 2172-2175.

16. Kim, J.; Lee, G.-H.; Jung, W.; Hah, S. S. Biosens. Bioelectron 2013, 43, 362-365.

17. Mortz, E.; Krogh, T. N.; Vorum, H.; Görg, A. Proteomics 2001, 1, 1359-1363. 